\title{
Experimental Investigation of Thermal Strain Caused by Electrical Discharge Machining on Stainless Steel SUS430
}

\author{
Chao-Ching Ho, ${ }^{*}$ Yuan-Jen Chang, ${ }^{1}$ Jin-Chen Hsu, ${ }^{1}$ Chia-Lung Kuo, ${ }^{1}$ and Fu-Chen Huang ${ }^{1}$ \\ Graduate Institute of Manufacturing Technology and Department of Mechanical Engineering, \\ National Taipei University of Technology, 1, Section 3, Zhongxiao E. Rd., Taipei 10608 Taiwan, R.O.C. \\ ${ }^{1}$ Department of Mechanical Engineering, National Yunlin University of Science and Technology, \\ 123 University Road, Section 3, Douliou, Yunlin 64002, Taiwan, R.O.C.
}

(Received May 1, 2017; accepted September 14, 2017)

Keywords: electrical discharge machining (EDM), digital image correlation (DIC), thermal strain, stainless steel SUS430

In this work, an experimental investigation of thermal strain caused by electrical discharge machining on a stainless-steel (SUS430) plate using digital image correlation is proposed. A speckle pattern was prepared by grinding the surface of objects to obtain a reliable and accurate measurement. The potential of the proposed system for thermal strain measurement using digital image correlation, combined with the ring-core method for electrical discharge machining, was evaluated. Ring-core machining involves milling a ring core around the inside of a displacement field analyzed by digital image correlation. It was observed that the peak strain presented in the outer ring and the strain decreased inward toward the inner core. Experimental results revealed that the maximum thermal strain caused by electrical discharge machining was proportional to the drilling depth.

\section{Introduction}

Electrical discharge machining (EDM) drilling has attracted considerable interest from the precision machining industry owing to its wide range of industrial applications and the advantage of having no constraints when machining ferrous materials. ${ }^{(1)}$ EDM drilling has demonstrated the ability to produce extremely precise holes in various metals. Since no physical contact occurs between the electrode and the workpiece materials, EDM is a noncontact and effective machining process that eliminates cutting forces. However, EDM causes thermally induced residual stress ${ }^{(2,3)}$ owing to the EDM arc plasma temperature, which ranges from $8000^{(4)}$ to $12000 \mathrm{~K}^{(5)}$ during the EDM process, necessary to exploit the melting and evaporation of workpiece materials. ${ }^{(6)}$ Each discharge removes some volume of material, and craters are generated on the surface. Hence, the EDM process is based on electrothermal mechanisms where a series of discrete discharges between the electrode and the workpiece material are generated.

Therefore, the EDM process also creates additional residual stress owing to rapid heat transfer from the high-temperature electric spark plasma and the fast quenching mechanism of the dielectric. ${ }^{(7)}$ As a result of the generation of additional thermally induced residual stresses that form in the top layer of a machined surface, the EDM process can cause major deformation of the workpiece. The

*Corresponding author: e-mail: hochao@ntut.edu.tw

http://dx.doi.org/10.18494/SAM.2017.1735

ISSN 0914-4935 @ MYU K.K. 
state of thermally induced residual stress affects the fatigue and stability of a machined component. ${ }^{(8)}$ Hence, it is necessary to predict the level of strain induced by the EDM process. To obtain more insight into the EDM processes, it is important to determine the induced thermal strain after the discharge process.

Thermal residual strain measurement is based on stress relaxation when eliminating materials containing thermal residual stresses. Stress relaxation causes a new equilibrium of forces and moments to form, which results in the deformation of the workpiece. This deformation is measured to determine the induced thermal residual strain after EDM. However, the traditional strain gauges contact with the material and work in a limited temperature range. ${ }^{(9)}$ Furthermore, the thermal residual strain of the surfaces can be examined by scanning electron microscopy (SEM) ${ }^{(10)}$ X-ray diffraction (XRD), ${ }^{(11)}$ and Raman spectroscopy ${ }^{(12)}$ to study the behavior of material under thermal stress. However, the disadvantages of these methods are their high cost and limited applicability to field use.

Digital image correlation (DIC) is a noncontact full-field measurement of thermal deformations on the surfaces of objects. ${ }^{(13,14)}$ In our previous studies, we presented a digital image correlation technique in combination with laser hole drilling to measure the residual thermal strain produced by laser milling for the aluminum alloy A15052 under plane strain tension conditions. ${ }^{(15)}$ Fullfield in-plane deformation fields associated with machining changes were measured by the DIC technique. Therefore, it was shown that DIC can be used directly to quantitatively determine the deformation field thermal strain.

To the best of our knowledge, optic noncontact measurements and DIC algorithms to determine the thermal strain caused by EDM have not been used. The aim of this study was to use electrical discharge machining on a stainless-steel (SUS430) plate and combine the ring-core method to characterize the induced residual thermal strain by DIC.

\section{Speckle Pattern for DIC Method}

DIC is an image-based method that measures the thermal deformation and strain fields on the surface of a workpiece undergoing electrical discharge machining. DIC is a full-field optical method and provides data-rich maps of thermal strain. However, the quality of the speckle pattern under high-temperature EDM strongly affects the performance of measurements made using DIC. The speckle pattern itself must be able to withstand the machining temperatures.

The speckle pattern designed for microscale DIC must be able to follow the deformation of the specimen, avoid the transformation of the patterning material itself, and prevent a reaction between the patterning material and specimen. Therefore, the boundary between the speckle pattern and the material surface must remain intact during deformation. ${ }^{(16)}$

Solutions with bare material surfaces ${ }^{(9,17,18)}$ have been proposed to overcome this issue instead of applying a coating ${ }^{(19)}$ or using paint deposition. ${ }^{(20)}$ By using the natural local features produced from bare material surfaces, the displacement can be directly calculated from the surface features without special surface preparation. For this purpose, grit-400 sandpaper is used to manually grind the bare material surface, and the variable gray intensity pattern can be generated at the material surface owing to the different levels of optically rough scattering, ${ }^{(21)}$ in contrast to a speckle pattern generated by painting the surface. ${ }^{(9)}$ The change in contrast was used as a random pattern and the speckle pattern acted as the carrier of surface deformation. Additionally, the ground surface of the sample was benefited by the removal of any residual surface damage. 
The images acquired by optical microscopy at a magnification of $4.06 \mu \mathrm{m} /$ pixel with a size of $640 \times 480$ pixels could be incorporated with DIC to measure the thermal strain distribution. The area of the pattern captured for DIC analysis was $2.6 \times 1.9 \mathrm{~mm}^{2}$. Figure 1 shows the speckle image of the specimen surface after being ground. A square area with a size of $200 \times 200$ pixels in the middle of the specimen was chosen to be the region of interest (ROI) for DIC analysis.

\section{Experiments and Discussion}

The scheme of the experimental setup of the proposed EDM system is presented in Fig. 2. Defining each discharge in the EDM process as axisymmetric allows for the assumption that the residual stresses in two perpendicular directions are equal. To examine the residual strain within a workpiece induced by EDM, the specimens were annealed before performing the EDM hollow hole drilling. Before the DIC measurement, the prepared workpieces were ultrasonically cleaned using ethanol to remove any contamination on the surface. The speckle patterns were acquired using a commercial DIC system. The area of the pattern acquired for DIC analysis was approximately $0.82 \times 0.82 \mathrm{~mm}^{2}$. The machining workpieces were prepared by sanding the surface with 400 -grit paper. Their performance as DIC deformation carriers and their stability during high-temperature EDM were examined in our previous work. ${ }^{(22)}$

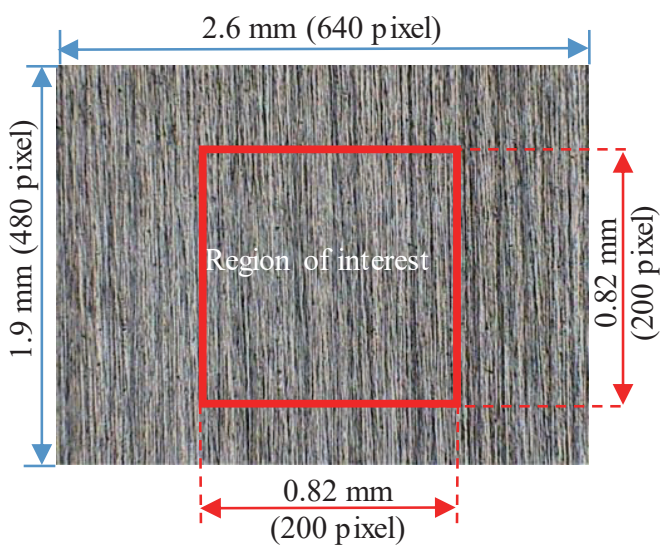

Fig. 1. (Color online) Photograph of ROI showing pattern produced on surface of specimen using grit paper. The highlighted rectangular area of $0.82 \times 0.82$ $\mathrm{mm}^{2}$ indicates the ROI for DIC analysis.

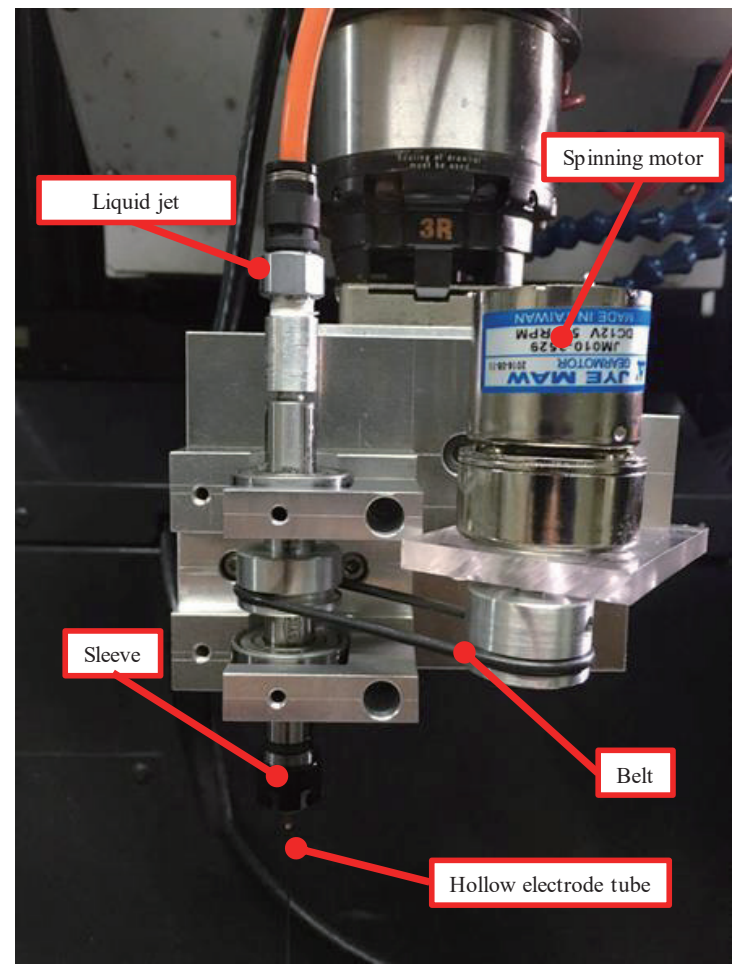

Fig. 2. (Color online) Setup for measuring thermal strain by EDM. 
The entire experimental system was placed on the stage of a Sodick AP1L Micro Precision electrical discharge machining machine (Sodick, Japan). All tests were done on steel (SUS430). The samples had a length of $25 \mathrm{~mm}$, a width of $25 \mathrm{~mm}$, and a thickness of $1 \mathrm{~mm}$. The diameter of the drilled hole, $D$, was $2 \mathrm{~mm}$. To identify the strain released by EDM, a hollow hole was drilled at the center of the workpiece. Finally, kerosene was chosen as the dielectric liquid, and coppertungsten $(\mathrm{Cu}-75 \mathrm{wt} \% \mathrm{~W})$ was used as the electrode material owing to its favorable wear resistance properties. The machining time was recorded from the start of drilling the specific region to a series of different depths under a discharge capacitance of $0.007 \mu \mathrm{F}$. Furthermore, images from an optical microscope were acquired using digital cameras for DIC analysis. An optical microscope with a $2.5 \times$-magnification lens was used.

As shown in Fig. 3, the machining process is a combination of a spinning hollow electrode tube and a liquid jet with a pressure of $0.1 \mathrm{MPa}$ injected through the hollow electrode tube automatically. The hollow electrode tube was clamped to a sleeve and was transported through a belt to the spinning motor. The spinning motor rotated at a defined rotational speed. The spinning process suppressed the nonuniform thermal distribution between the tube and the machining specimen. The liquid jet helped to flush the debris and avoid debris concentration.

Owing to this process setup, different ring core sizes can be realized with variable tube diameters. For metallographic observations, the depth of EDM was measured after 2 to $3 \mathrm{~h}$ of careful polishing along the vertical direction of the specimen until the machined groove became visible. The cross sections of the ring cores were carefully checked to determine the accurate profile of machining. The depth and diameter of the machined ring core groove were investigated by optical microscopy (Olympus U-PMTVC 8C14561). The profile image and the inner and outer ring diameters after EDM are shown in Fig. 4.

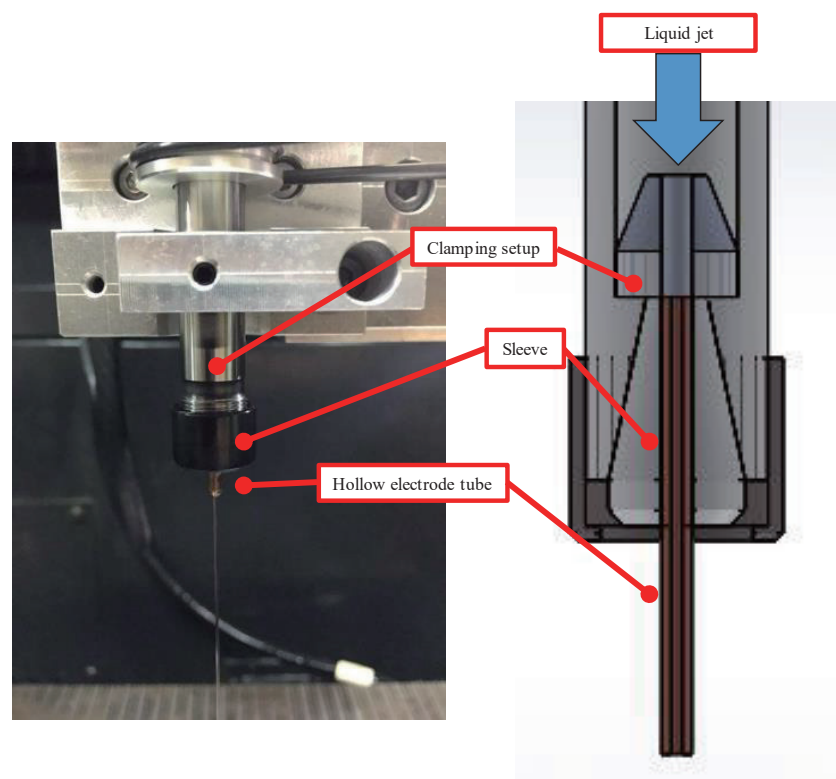

Fig. 3. (Color online) Combination of spinning and liquid jet for tube EDM. 


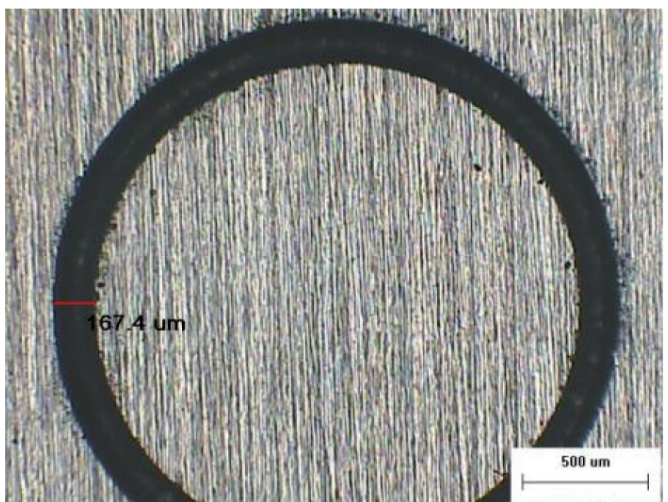

(a)

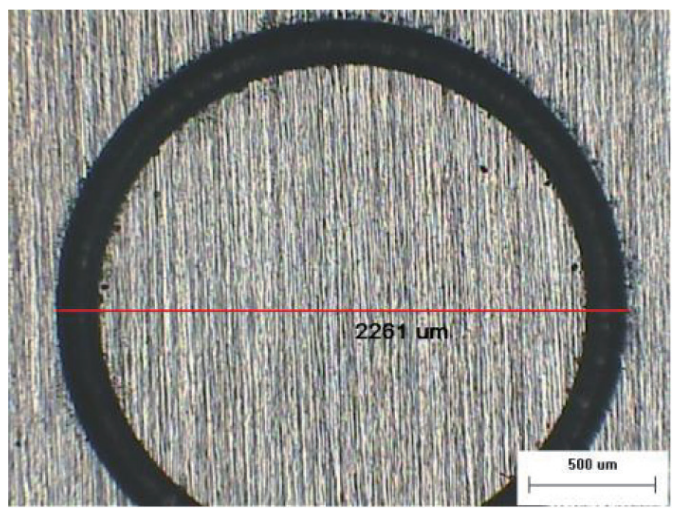

(c)

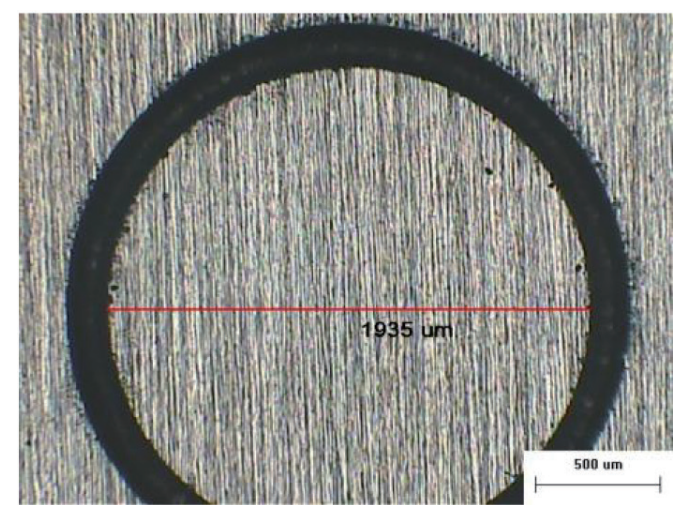

(b)

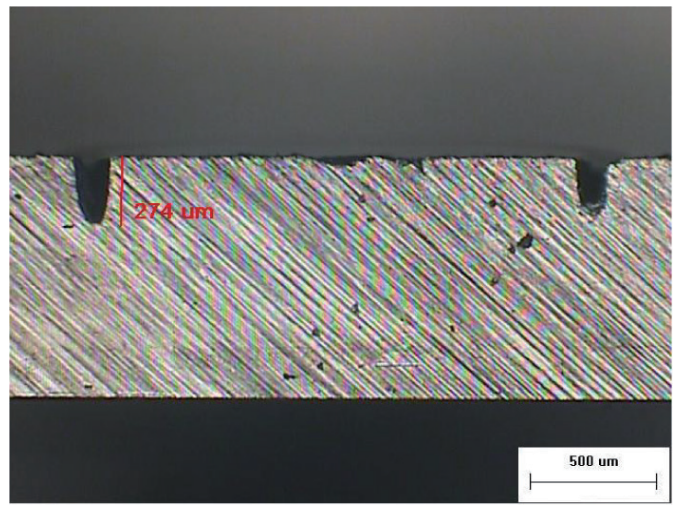

(d)

Fig. 4. (Color online) Optical microscopy images $(25 \times)$ of machined ring core groove: (a) groove width, (b) inner diameter, (c) outer diameter, and (d) depth.

To investigate the thermal strain induced by the EDM, a ring-core holes of different depths (i.e., 220, 430, and $650 \mu \mathrm{m}$ ) were machined in the surface of the specimen, as shown in Fig. 5.

The results are shown in Figs. 6-8, which demonstrate the thermal strain field induced by EDM under the working parameters of $105 \mathrm{~V}, 0.007 \mu \mathrm{F}, 3 \mu \mathrm{s}$, and $6 \mu \mathrm{s}$ (open circuit voltage, discharge capacitance, pulse-on duration, and pulse-off duration, respectively) and the colored area representing the ROI where the process was carried out. The EDM conditions are listed in Table 1. Strain fields were computed using VIC-2D, a commercially available DIC algorithm produced by Correlated Solutions. The DIC patches $(51 \times 51$ pixels overlapped by $75 \%)$ covered the entire imaged area apart from the inward and outward contours of the ring and its immediate vicinity, where a thermal recast layer was left over by the EDM process.

As depicted in Fig. 9, the experimental results showed that the peak thermal strain induced in SUS430 by EDM hole drilling was approximately proportional to the drilling depth. It is observed that the peak strain is present in the outer ring, and the strain decreases inward toward the inner core. The experimental results show good agreement with those presented in the literature. ${ }^{(2,3)}$ During the EDM process, the machined outer ring surface is exposed to plasma with a high temperature. The EDM process can cause major deformation in the outward area of the ring 


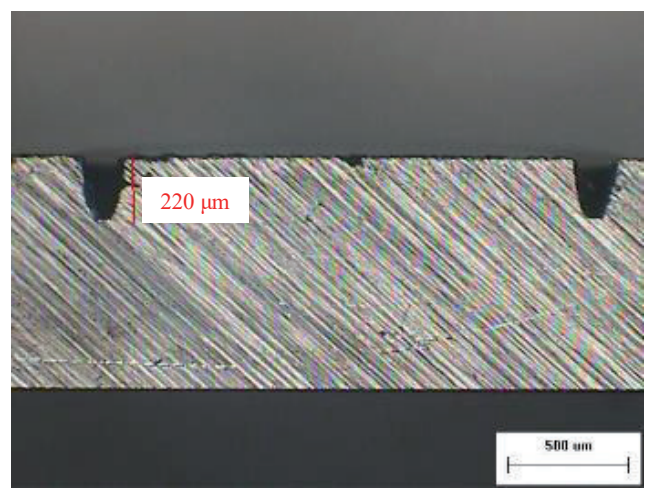

(a)

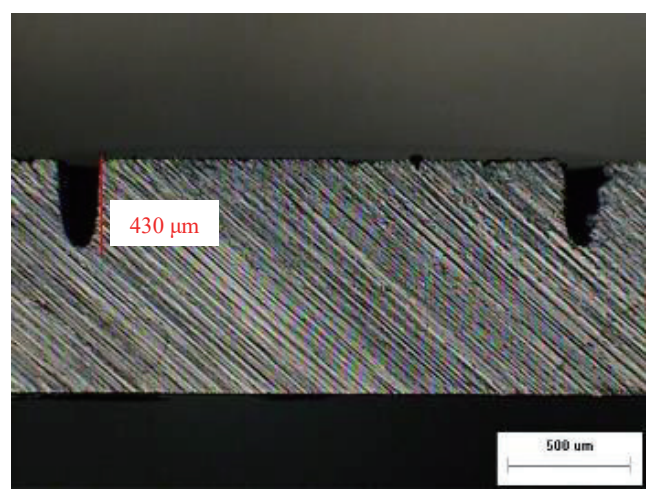

(b)

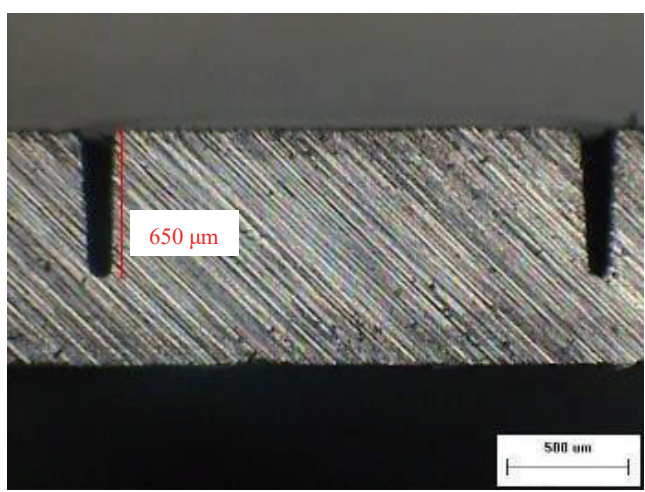

(c)

Fig. 5. (Color online) Profile images (25×) of machined ring core groove at different machined depths: (a) 220, (b) 430 , and (c) $650 \mu \mathrm{m}$.

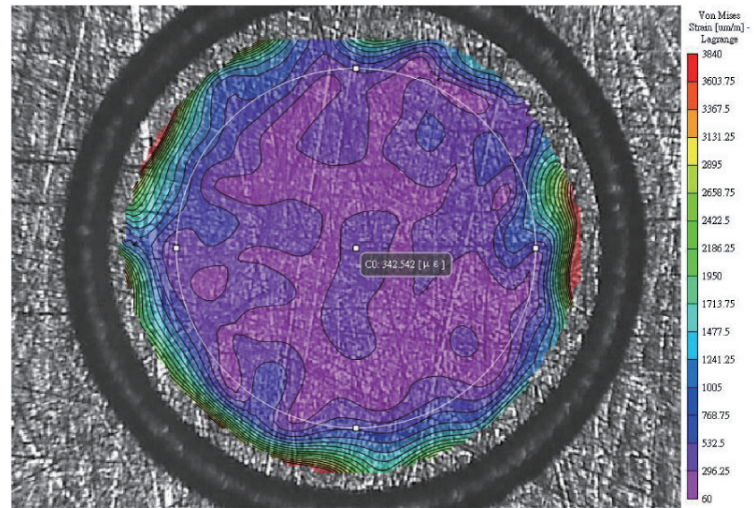

Fig. 6. (Color online) Strain induced by EDM at a depth of $220 \mu \mathrm{m}$. The average strain obtained by the 2D-DIC measurement matching process in the ROI was $343 \mu \varepsilon$. The maximum strain of $3840 \mu \varepsilon$ occurred at the rim of the core.

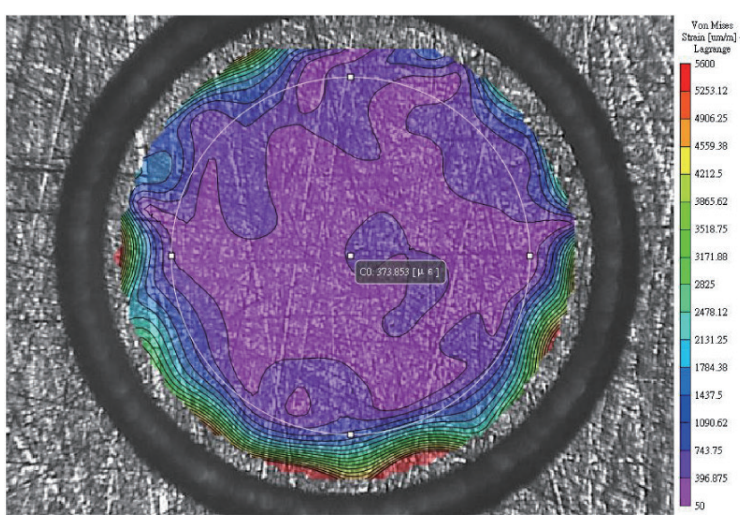

Fig. 7. (Color online) Strain induced by EDM at a depth of $430 \mu \mathrm{m}$. The average strain obtained by the 2D-DIC measurement matching process in the ROI was $374 \mu \varepsilon$. The maximum strain of $5600 \mu \varepsilon$ occurred at the rim of the core. 


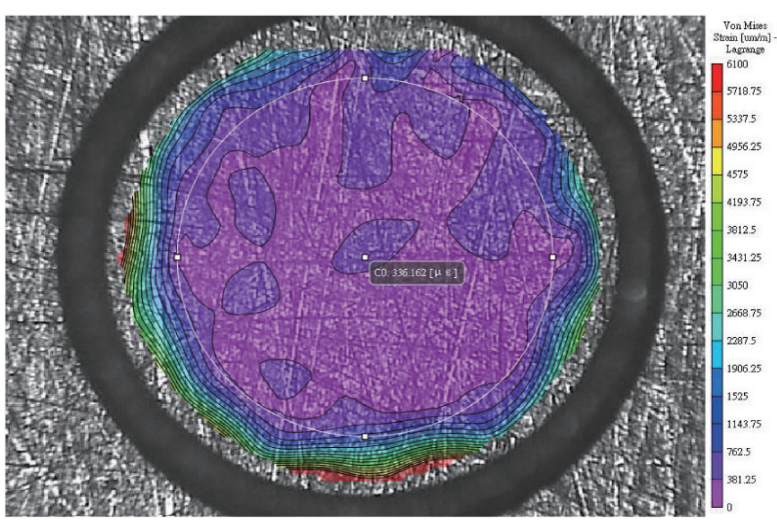

Fig. 8. (Color online) Strain induced by EDM at a depth of $650 \mu \mathrm{m}$. The average strain obtained by the 2D-DIC measurement matching process in the ROI was $336 \mu \varepsilon$. The maximum strain of $6100 \mu \varepsilon$ occurred at the rim of the core.

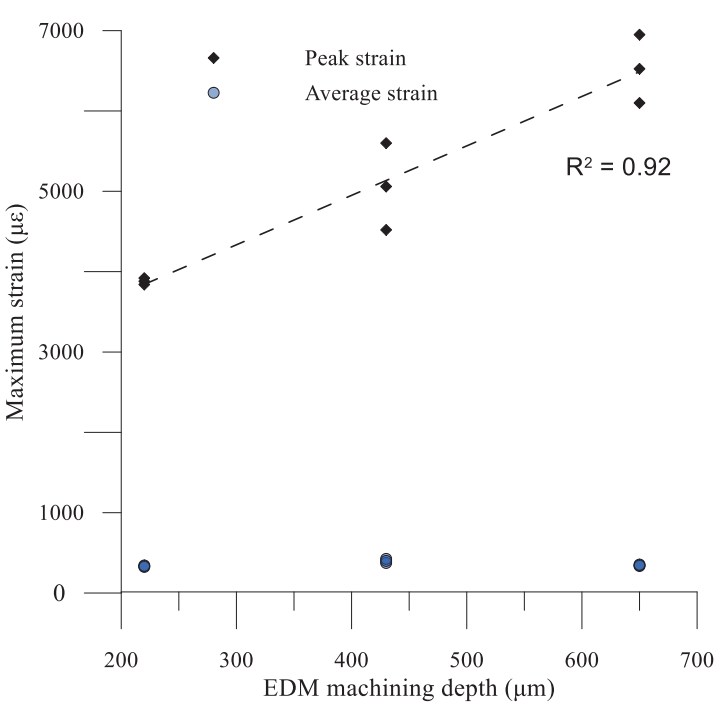

Fig. 9. (Color online) Peak and average strains induced by EDM at different machining depths.

Table 1

EDM conditions used for experimental tests.

\begin{tabular}{lc}
\hline \multicolumn{2}{c}{ EDM conditions } \\
\hline On-time & $3 \mu \mathrm{s}$ \\
Off-time & $6 \mu \mathrm{s}$ \\
Open-circuit voltage & $105 \mathrm{~V}$ \\
Discharge capacitance & $0.007 \mu \mathrm{F}$ \\
Electrode material & Copper \\
Dielectric fluid & Kerosene \\
Flushing pressure & $0.1 \mathrm{MPa}$ \\
\hline
\end{tabular}

sections. However, the strain deformation is slightly small after a penetration depth of $200 \mu \mathrm{m}$ below the surface. Meanwhile, this is observed for different machined depths given that the average strains obtained using a 2D-DIC measurement matching process within the ROI (i.e., white circular line) were similar. This result exhibits the advantage of the ring-core method that enables the measurement of the profile of the residual strain in a material by advanced optical sensing with high sensitivity.

\section{Conclusions}

Because EDM is a thermal machining process, the characterization of additional thermal strain induced by the rapid chilling contraction is very important. The utilization of the pattern produced by grinding the surface resulted in a satisfactory strain deformation measurement under EDM. In this paper, we presented the results of a series of machined depth experiments to explore the 
capability of digital image correlation to measure thermal strain distributions under EDM. This work highlighted the essential requirements for understanding the degree of thermal strain induced by the EDM process. Future work will extend the on-line sensing of residual strain by the ringcore method with the EDM hollow hole-drilling operation.

\section{Acknowledgments}

This work was supported by the Ministry of Science and Technology, Taiwan, R.O.C., MOST 106-2622-E-027-014-CC3 and 106-2221-E-027-051.

\section{References}

1 S.-H. Kang, T.-H. Fang, L. Riccobono, and G. Carbone: Smart Science 1 (2013) 69.

2 J.-P. Kruth and P. Bleys: Int. J. Electr. Mach. 5 (2000) 23.

3 S. Saxena: Int. J. Sci. Eng. Res. 7 (2016) 165.

4 K. Albinski, K. Musiol, A. Miernikiewicz, S. Labuz, and M. Malota: Plasma Sources Sci. Technol. 5 (1996) 736.

5 H. Yoshida and M. Kunieda: Int. J. Jpn Soc. Precis. Eng. 62 (1996) 1464.

6 J. A. Sánchez, B. Izquierdo, N. Ortega, I. Pombo, S. Plaza, and I. Cabanes: Int. J. Comput. Integr. Manuf. 22 (2009) 799.

7 H.-T. Lee and F.-C. Hsu: Mater. Sci. Technol. 19 (2003) 1261.

8 N. Rossini, M. Dassisti, K. Benyounis, and A. Olabi: Mater. Des. 35 (2012) 572.

9 R. B. Berke and J. Lambros: Rev. Sci. Instrum. 85 (2014) 045121.

10 Y. H. Jia, J. G. Li, and X. J. Lu: Adv. Mater. Res. 68 (2011) 309.

11 N. Ferreira, E. Abramof, E. Corat, N. Leite, and V. Trava-Airoldi: Diamond Relat. Mater. 10 (2001) 750.

12 Z. Nibennaoune, D. George, F. Antoni, S. Ahzi, D. Ruch, J. Gracio, and Y. Remond: Diamond Relat. Mater. 31 (2013) 81.

13 G. Stoilov, V. Kavardzhikov, and D. Pashkouleva: J. Theor. Appl. Mech. 42 (2012) 55.

14 L.-C. Chen, C.-Y. Chang, W.-C. Lee, and C.-C. Ma: Smart Sci. 3 (2015) 80.

15 C.-C. Ho, Y.-J. Chang, J.-C. Hsu, C.-L. Kuo, and G.-H. Lee: Sens. Mater. 28 (2016) 1067.

16 J. Quinta da Fonseca, P. Mummery, and P. Withers: J. Microsc. 218 (2005) 9.

17 B. K. Bay: J. Orth. Res. 13 (1995) 258.

18 B. Grant, H. Stone, P. Withers, and M. Preuss: J. Strain Anal. Eng. Des. 44 (2009) 263.

19 Y. Dong, H. Kakisawa, and Y. Kagawa: Opt. Lasers Eng. 68 (2015) 7.

20 G. Lionello and L. Cristofolini: Meas. Sci. Technol. 25 (2014) 107001.

21 C. Sebastian and E. Patterson: Exp. Tech. 39 (2015) 21.

22 C.-C. Ho, D.-S. Wu, Y.-J. Chang, J.-C. Hsu, C.-L. Kuo, and S.-K. Kuo: J. Laser Micro/Nanoeng. (in press).

\section{About the Authors}

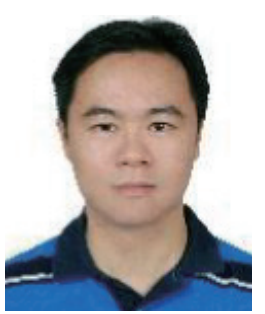

Chao-Ching Ho received his B.S. and M.S. degrees from National Taiwan University, Taipei, Taiwan, R.O.C., in 1995 and 1997, respectively, and his Ph.D. degree in electrical engineering from the National Taiwan University of Science and Technology, Taipei, Taiwan, in 2008. From 2009 to 2016, he was with the Department of Mechanical Engineering, National Yunlin University of Science and Technology, where he was an associate professor. Since 2016, he has been with the Graduate Institute of Manufacturing Technology, National Taipei University of Technology, where he is currently an associate professor. His research interests are metrology, prognostic system and laser ablation, as well as machine vision. 


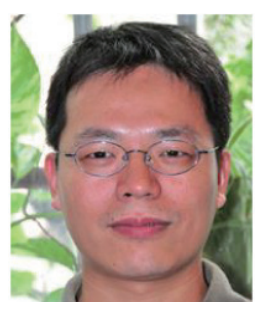

Yuan-Jen Chang received his B.S. and M.S. degrees from National Taiwan University and National Cheng Kung University, Taiwan, in 1996 and 1998, respectively. He received his Ph.D. degree from the University of Colorado at Boulder in 2008. From 2009 to 2013, he was an assistant professor at the Department of Mechanical Engineering, National Yunlin University of Science and Technology, where he is currently an associate professor. His research interests are nanoimprinting, nanofabrication technologies, and laser material processing.

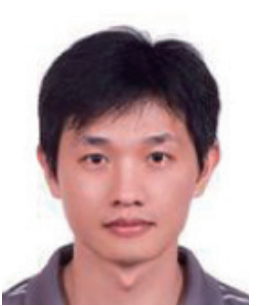

Jin-Chen Hsu received his B.S. degree in mechanical engineering from the National Taiwan University of Science and Technology in 1997 and his Ph.D. degree in applied mechanics from the National Taiwan University in 2007. From 2007 to 2009, he was a postdoctoral research fellow at the Institute of Applied Mechanics of the National Taiwan University. He joined the faculty of the Department of Mechanical Engineering, National Yunlin University of Science and Technology, Taiwan, and currently, he is an associate professor. His research interests include acoustic waves in piezoelectric media and metamaterials, finite-element simulations, and laser machining.

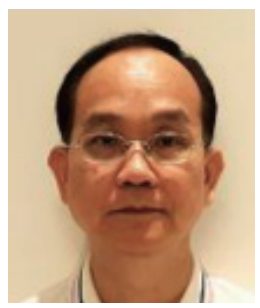

Chia-Lung Kuo received his B.S. degree from National Chung Hsing University, Taiwan, R.O.C., in 1984 and his M.S. and Ph.D. degrees from the University of Tokyo, Japan, in 1989 and 1992, respectively. From 1992 to 2010, he was an associate professor at the Department of Mechanical Engineering, National Yunlin University of Science and Technology, Taiwan. Since 2010, he has been a professor at the same university. His research interests are in EDM, $\mathrm{ECM}$, and laser processing.

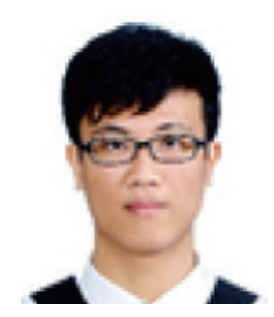

Fu-Chen Huang received his B.S. degree from Far East University, Taiwan, R.O.C., in 2016, and he is currently a graduate student in the Department of Mechanical Engineering, National Yunlin University of Science and Technology, Taiwan. His research interests are in laser micromachining and EDM. 